Check for updates

Cite this: Chem. Sci., 2019, 10, 4822

๑ All publication charges for this article have been paid for by the Royal Society of Chemistry

Received 9th February 2019

Accepted 28th March 2019

DOI: $10.1039 / c 9 s c 00704 k$

rsc.li/chemical-science

\section{A microdroplet-accelerated Biginelli reaction: mechanisms and separation of isomers using IMS- MS†}

\author{
Navneet Sahota, (D) \$ Deyaa I. AbuSalim, D $\$$ Melinda L. Wang, Christopher J. Brown, \\ Zhicaho Zhang, Tarick J. El-Baba, (D) Silas P. Cook (D)* and David E. Clemmer (D)*
}

Electrospray ionization (ESI) combined with ion mobility spectrometry (IMS) and mass spectrometry (MS) techniques is used to examine the Biginelli reaction in an ensemble of ions generated from droplets. We find evidence for rapid dihydropyrimidinone formation from condensation of ethyl acetoacetate, benzaldehyde, and urea on the very short timescales associated with the electrospray process ( $\sim 10 \mu$ s to $\sim 1.0 \mathrm{~ms}$ ). Control bulk-solution reactions show no product formation even after several days. This implies that the in-droplet reaction rate is enhanced by an astonishing factor. Examination of the reaction conditions and characterization of the intermediates en route to product shows evidence for variations in the reaction mechanism. IMS separation shows that the Knoevenagel condensation intermediate from benzaldehyde and ethyl acetoacetate exists as both the cis- and trans-isomer, in a $\sim 5$ to 1 ratio. We suggest that the dramatic acceleration arises because of increased reagent confinement as electrosprayed droplets shrink. The ability of IMS-MS to resolve intermediates (including isomers) provides a new means of understanding reaction pathways.

\section{Introduction}

In his 1893 seminal report, Pietro Biginelli showed that benzaldehyde (1) can be condensed with ethyl acetoacetate (2) and urea (3) to form dihydropyrimidinone (4) in high yield (Scheme 1). ${ }^{1}$ This transformation created a foundational multicomponent reaction (MCR) that changed the way chemists thought about constructing molecules. MCRs offer access to greater chemical diversity and are more efficient than other multi-step bimolecular processes. ${ }^{2}$ With its inherent modularity, ${ }^{3,4}$ the Biginelli reaction has unlocked vast libraries of dihydropyrimidinones, ${ }^{5}$ dihydropyrimidinthiones, ${ }^{6}$ and guanidines. ${ }^{7}$ Furthermore, the reaction has enabled the synthesis of both racemic $^{6}$ and enantioenriched ${ }^{8-11}$ molecules that exhibit significant anti-cancer, anti-microbial, anti-inflammatory, antiviral, and calcium-channel inhibitory properties. ${ }^{6}$

While the Biginelli reaction proceeds with a wide range of catalysts, including Lewis acids, Brønsted acids, hydrogen-bond donors, and even transition metals, ${ }^{12}$ attempts to realize a catalyst-free Biginelli reaction ${ }^{13-15}$ have been met with mixed success. ${ }^{16}$ The development of a mild, room temperature

Department of Chemistry, Indiana University, 800 East Kirkwood Avenue, Bloomington, IN 47405-7102, USA. E-mail: clemmer@indiana.edu; sicook@indiana. edu

$\dagger$ Electronic supplementary information (ESI) available. See DOI: 10.1039/c9sc00704k

\$ N. S. and D. I. A. contributed equally to this work. protocol for the production of dihydropyrimidinones remains an important goal in the field. Such reactions offer the possibility of greater substrate scope and new reaction development.

Recently, the groups of Cooks, ${ }^{17-26}$ Zare $^{27-39}$ and others ${ }^{\mathbf{4 0 - 4 6}}$ have reported the acceleration of reactions in microdroplet environments during the process of electrospraying reagents into a mass spectrometer. ${ }^{32}$ Intrigued by the possibility of faster rates for multicomponent reactions, we explored the use of microdroplets as a means of enabling a rapid Biginelli reaction (Scheme 2). Here, we describe an extraordinary rate enhancement en route to Biginelli product 4 using only microdroplet evaporation. To date, the only other MCR that has been observed on microdroplets is the Hantzsch synthesis (which showed a rate enhancement of $\left.\sim 10^{5}\right) .{ }^{23}$ The ability to study MCRs on microdroplets is potentially paradigm shifting. Reagent confinement may allow many new reaction mechanisms to be accessed and monitored with the ease and power of mass spectrometry (MS). The ability to detect, separate, and<smiles>CCOC(=O)CC(C)=O</smiles>

Scheme 1 The condensation of benzaldehyde (1), ethyl acetoacetate (2), and urea (3) leading to the Biginelli synthesis of dihydropyrimidinones (4). 


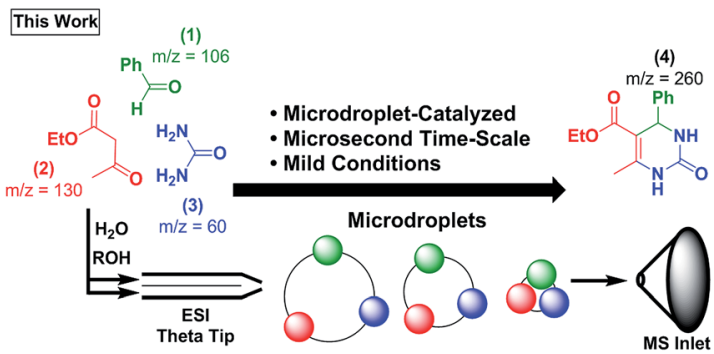

Scheme 2 Our report of the microdroplet-catalyzed Biginelli reaction using theta tip.

alter the relative abundance of isomeric intermediates using ion mobility spectrometry (IMS) combined with MS is a first for microdroplet chemistry. ${ }^{47,48}$

\section{Result and discussion}

Rate acceleration relative to bulk-phase control reactions

In the bulk-phase, refluxing 1,2 , and 3 in methanol and ethanol, in the presence of catalytic $\mathrm{HCl}$, for 2 hours resulted in the precipitation of the Biginelli product 4 (Scheme 3a). Yet, several reports of heating 1, 2, and 3 in the absence of exogenous catalyst in acetonitrile, ${ }^{49,50}$ ethanol, ${ }^{51}$ octane, ${ }^{52}$ ethylene glycol, ${ }^{53}$ or neat fails to produce Biginelli product 4 (Scheme 3b). ${ }^{54}$ Additionally, we performed an uncatalyzed, room temperature reaction that only produced trace amounts of $\mathbf{4}$ after 48 hours of stirring (see ESI $\uparrow$ for details).

Our results indicate that the Biginelli reaction is accelerated in microdroplets produced by electrospray ionization (ESI). For this a solution of $\mathbf{1}, 2$, and 3 in $1: 1 \mathrm{MeOH}: \mathrm{H}_{2} \mathrm{O}$ was sprayed as a plume of charged droplets, and the resulting mixture analysed by MS (see ESI $\dagger$ for experimental details). Product 4 was detected instantly. Since this reaction produces only trace products in methanol (see ESI $\dagger$ ) and acetonitrile at 48 hours, ${ }^{55}$ the microdroplet-catalyzed variant represents a significant rate enhancement. To quantify the rate enhancement, we used a theta-glass, double-barrel, capillary where a mixture of two reagents (e.g., 1 and 2) was sprayed from the first barrel, and the remaining reagent (e.g., 3) was sprayed from the second barrel (Fig. S1 $\dagger$ ). All combinations of reagents were analysed (see ESI $\dagger$ for details).

This method of spraying ESI generated droplets has been shown to allow fast mixing, on the order of $\mu$ s to ms during the ESI process. ${ }^{45}$ The observed product formation confirms that the reaction occurs on these short $\sim 10 \mu \mathrm{s}$ to $\sim 1 \mathrm{~ms}$ time-scales in microdroplets. This represents a massive acceleration relative to control bulk-phase reactions. ${ }^{55}$ This acceleration presumably arises as the microdroplet evaporates, a process that leads to high concentrations of reagents in confined spaces. Additional factors such as change in $\mathrm{pH},{ }^{27,28}$ droplet size, ${ }^{28,29}$ reagent concentration, ${ }^{28,34,46}$ solvent system, ${ }^{17,30}$ and droplet temperature, ${ }^{30,31}$ may also influence these reactions.

Based on recent reports, ${ }^{56,57}$ control experiments were needed to rule out gas-phase ion molecule reactions or clustering at the inlet. Indeed, the reaction did not proceed when

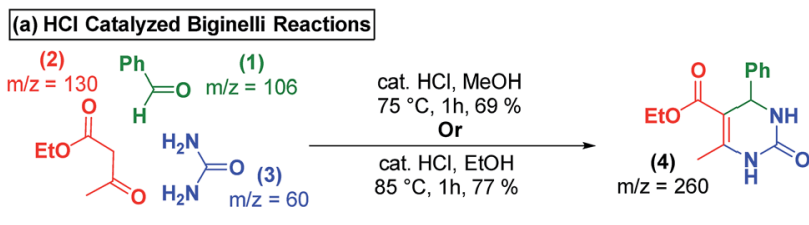

(b) Previously Reported Biginelli Reaction Attempts

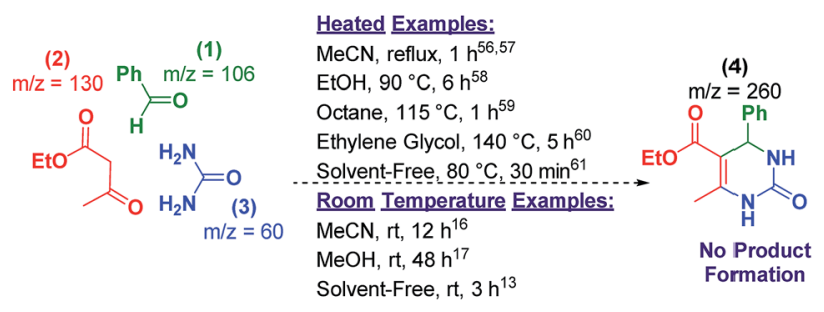

Scheme 3 (a) Acid-catalyzed Biginelli produced products in high yields. (b) Prior reports show that no reaction takes place at roomtemperature in the absence of a catalyst.

molecular benzaldehyde (1) component was introduced into the sheath gas while the other two reactants were electrosprayed (see ESI $\dagger$ ). Moreover, clustering at the inlet was ruled out by the efficiency with which the MS can thermalize ions, showing few if any buffer clusters, and only resolved ions. Taken together, these experiments support reaction in the droplets.

Comparison of data between spraying from a theta tip (double barrel capillary) ${ }^{43,45,48}$ and a single-barrel capillary indicated that this reaction proceeds faster using a theta tip. Fig. 1 shows that product peaks (e.g., $m / z=261$ and 283) as well as the intermediate peaks (e.g., $m / z=191$ and 219) are more abundant when using a theta tip. This is attributed to the incredibly fast mixing times ( $\mu$ s scale) reported for theta tips. ${ }^{45,58}$

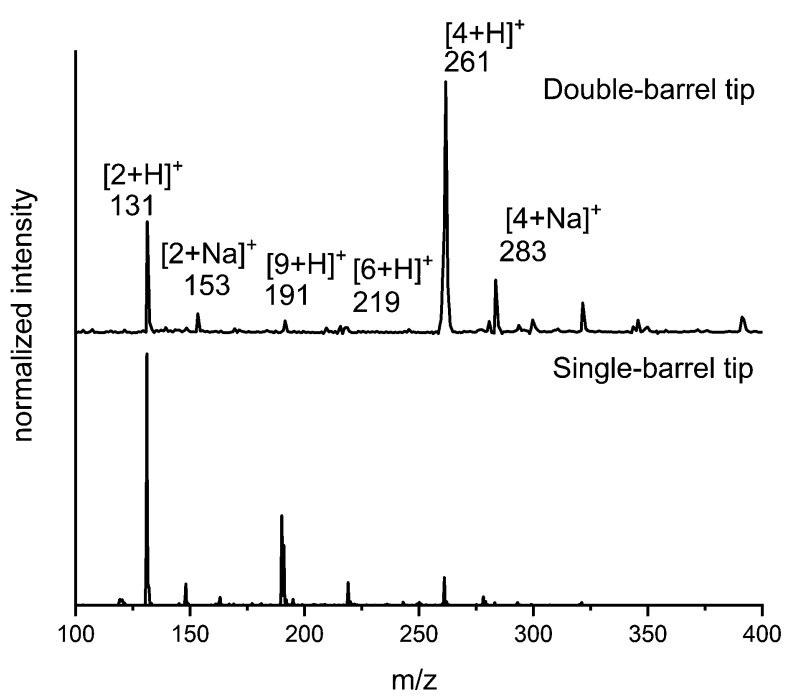

Fig. 1 ESI mass spectrum of the Biginelli reaction using a doublebarrel tip (top) and a single-barrel tip (bottom). In the double-barrel experiment, benzaldehyde (1) solution was sprayed from one barrel and a mixture of ethyl acetoacetate (2) and urea (3) were sprayed from the other barrel. 
The abundance of the product peak achieved after spraying the reagents using the single barrel tip under exact same conditions (see ESI† for details) is also shown in Fig. 1.

\section{Mechanistic insights for catalyst-free Biginelli in microdroplets}

Three mechanisms for the Biginelli reaction arise from the order in which the reagents 1,2 , and 3 can be combined to form 4. Additionally, the operative mechanism can be biased by the relative concentrations of the starting materials, and the conditions of this reaction., ${ }^{3,4}$ The first attempt at explaining this mechanism was reported by Folkers and Johnson in 1933. ${ }^{59}$ Four decades later, Sweet and Fissekis proposed an alternative wherein the reaction proceeds via acid-catalyzed aldol-condensation of benzaldehyde with ethyl acetoacetate to form an activated carbonium ion 6, which subsequently condenses with urea to form 4 (Scheme $4 a){ }^{60}$ Later an NMR-guided re-examination of the mechanism led Kappe to propose an iminium first pathway, wherein 4 is formed via an $N$-acyliminium ion 8 (Scheme $4 \mathrm{~b}$ ). ${ }^{61}$ While two of the proposed pathways involved benzaldehyde as the first reactant, Cepanec first proposed the condensation of urea with ethyl acetoacetate to form an enamine $\mathbf{1 0}$ from intermediate $\mathbf{9}$, which can then produce $\mathbf{4}$ after a condensation reaction with benzaldehyde (Scheme 4c). ${ }^{62}$ Cepanec's example differed from all previous postulates because the reaction proceeds in the presence of antimony(III) chloride, a Lewisacid. ${ }^{62}$ More recently, ${ }^{63}$ De Souze and coworkers used ESI-MS to study the intermediates of this reaction and identified the $\mathrm{N}$-acyliminium intermediate as expected from the mechanism proposed by Kappe, and also noted the presence of the bisurea intermediate detected by Folkers and Johnson. ${ }^{59,63}$ While several DFT studies have been reported on this reaction, they remain in conflict.6,63 Clearly, the observed mechanism depends on the reaction conditions and methods used to monitor and detect the intermediates.

With the unique reaction conditions explored in microdroplets, we were interested in probing the operative mechanism by analysing reaction intermediates by mass spectrometry.

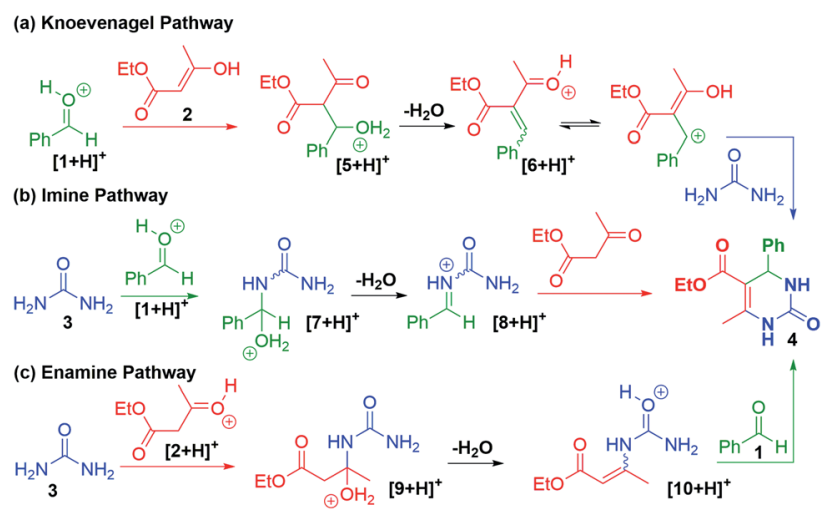

Scheme 4 Different pathways observed during investigations into the mechanism of the Biginelli reaction.
We detected intermediates with $\mathrm{m} / \mathrm{z}=191$ corresponding to intermediates along the enamine pathway (Scheme 4c). In addition, relatively small peaks for $\mathrm{m} / \mathrm{z}=219$ was detected which is an intermediate along the Knoevenagel mechanism (Scheme 4a). The data provided in Fig. 1 and 2 provide support for the presence of two competing pathways in microdroplets, the enamine and Knoevenagel pathways.

\section{Resolving isomeric intermediates using IMS-MS}

We have also examined the Biginelli reaction using our previously described IMS-MS techniques. ${ }^{\mathbf{6 4 , 6 5}}$ The simple Knoevenagel condensation can generate a mixture of isomers. IMS-MS allows these isomers to be separated prior to MS analysis. Fig. 2 shows the IMS separation for the $m / z=219$ ion. We resolved cis- and trans-isomer products formed upon condensation of benzaldehyde and ethyl acetoacetate, which are intermediates in synthesis of many biologically relevant molecules such as amino acids and antibiotics. These isomeric products are difficult to separate and characterize by traditional methods. Our assignments for the cis/trans isomers were made as follows: a bulk condensation of benzaldehyde and ethyl acetoacetate was used to produce these isomeric products, which are unstable at room temperature. These products were chromatographically separated, and NMR spectra were obtained to assign each isomer. Once assigned, these samples were used as standards to assign the isomer peaks separated by IMS. Our results indicate that the cis-isomer is favored in these reactions.

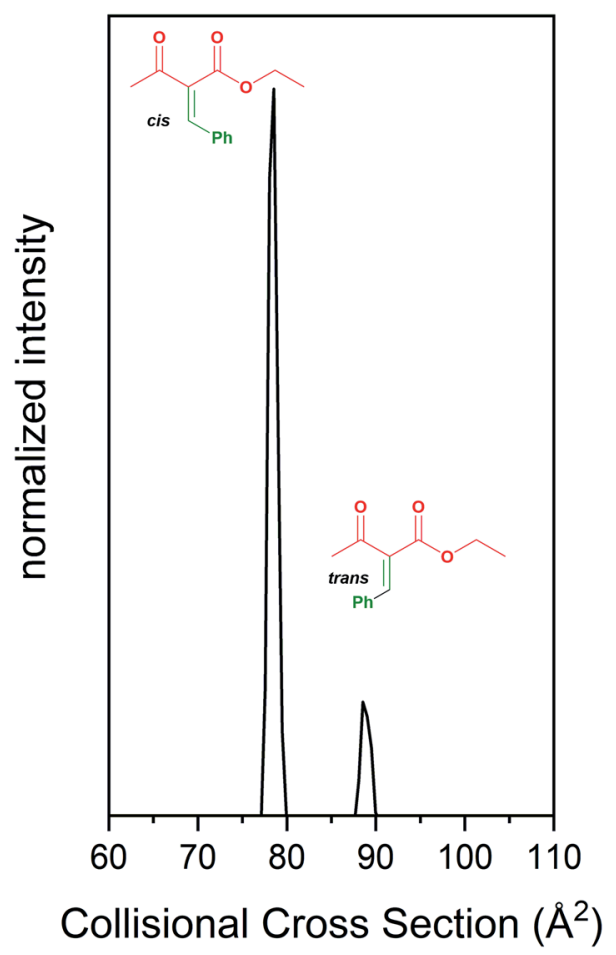

Fig. 2 Formation and separation of cis/trans isomers of an intermediate in the Biginelli reaction using IMS. 


\section{Temperature and solvent effects}

Based on previous reports, ${ }^{\mathbf{1 3 - 1 5}}$ we expected that an increase in the temperature of the electrosprayed droplets would alter the ratio of products and intermediates. To probe this effect, we carried out the in-droplet reaction while varying the temperature of the electrospray emitter from $5{ }^{\circ} \mathrm{C}$ to $65{ }^{\circ} \mathrm{C}$ (Fig. 3) using a variable temperature ESI source described previously. ${ }^{66}$ As temperature is increased, the abundance of the $\mathrm{m} / \mathrm{z}$

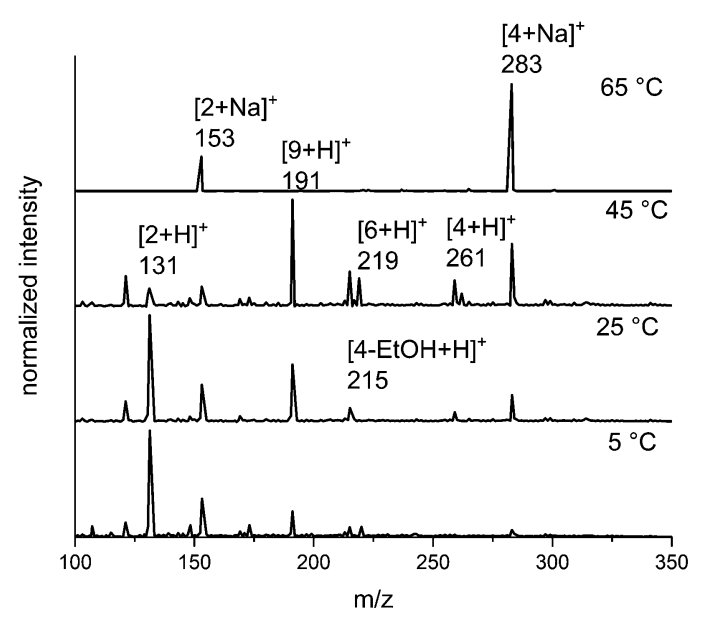

Fig. 3 The effect of temperature on the product distribution of the microdroplet-catalyzed Biginelli reaction. Elevated temperatures show higher product : intermediate ratios.
$=191,261$ and 283 ions increases, which corresponds a latestage intermediate and the products of the reaction. At the highest temperature, the products are favoured almost exclusively.

Finally, we note that the in-droplet Biginelli reaction also shows a strong solvent dependence. A series of systematic studies were performed by varying the solvent composition (e.g., different proportions of water, methanol, and ethanol). Aqueous mixtures of methanol or ethanol show more efficient product formation compared with pure methanol, ethanol and water. These results could be attributed to several factors. For example, changes in solvent will impact droplet size and the evaporative cooling rate. Interestingly, the predominant reaction mechanism was different when using various solvent systems. The major pathway in aqueous methanol mixtures follows the enamine pathway (Scheme 4c) as the intermediate peaks that were detected for $m / z=173$ and 191 were of higher intensity than those with $\mathrm{m} / z=261$ and 279 (Fig. 4). Whereas in aqueous ethanol, the mechanism shifts towards the Knoevenagel pathway (Scheme $4 \mathrm{a}$ ) as $\mathrm{m} / z=219$ and 261 are relatively more significant intermediates (Fig. 4). However, with water as a solvent 149 peak was observed next to $\mathrm{m} / \mathrm{z}=153$ that corresponded to the iminium pathway (Scheme $4 \mathrm{~b}$ ). The alteration in solvent system causes the alteration in the $\mathrm{pH}$ of the microdroplet system which further justifies that many factors play a role in microdroplet-catalysed reactions and routes the subsequent reagents take in the formation of the desired product. $^{33}$

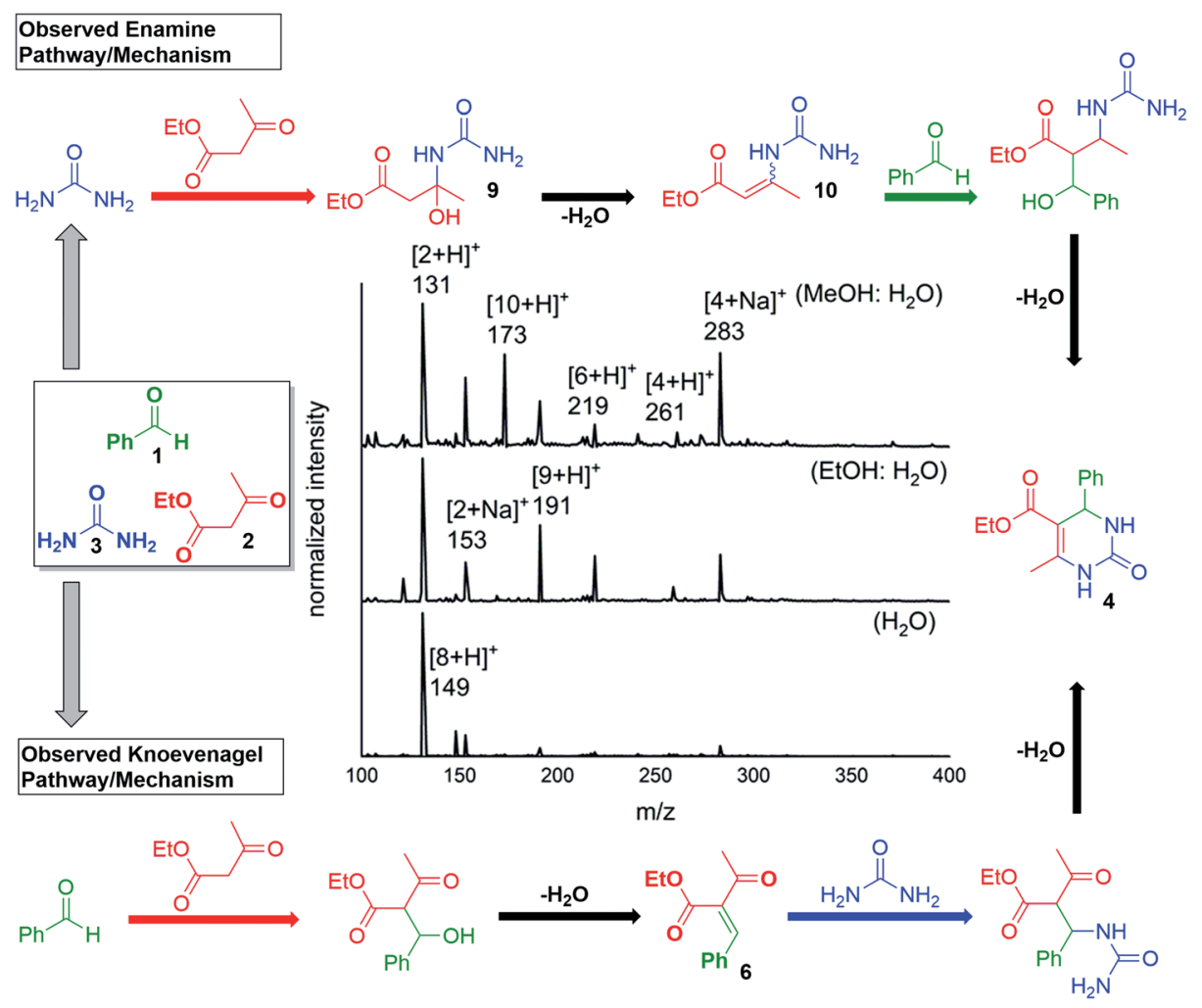

Fig. 4 The choice of solvent had a significant effect on the product distribution and conversion. $1: 1 \mathrm{MeOH}$ in water was found to be optimal. 
Occurrence of the same intermediates in the bulk-phase Biginelli reaction and the accelerated reaction occurring in droplets establishes the conflicting reports on reaction mechanism. Exploration of the solvent system, the concentration of the reagents (see $\mathrm{ESI} \dagger$ for details), and temperature effects for the electrospray reaction emphasizes the role that droplet desolvation and the corresponding energy provided to the droplet plays in reaction progress on the microsecond timescale.

\section{Conclusions}

An ESI-based microdroplet-catalyzed Biginelli reaction was developed. The reaction occurs on a microsecond timescale in microdroplets with a massive rate acceleration relative to the analogous bulk-phase Biginelli reaction. Our studies showed that a $1: 1$ methanol : water system gave the best conversion to products. Higher temperature, lower concentration of the reagents in the solution, smaller emitter tip sizes and theta $v s$. single barrel tips play role in the acceleration of the MCR and the pathway it undergoes to form the desired product. Offline collection of the product further solidified the importance of reactions happening in microdroplets.

\section{Experimental}

\section{Reagents}

All reagents including benzaldehyde, ethyl acetoacetate, urea, methanol, ethanol and deionized water were purchased from Sigma-Aldrich (Milwaukee, WI) and used without any further purification.

\section{Electrospray source}

Different experiments used the different electrospray ionization sources. For the rate acceleration studies theta tips were pulled to an inner diameter size of $\sim 6 \mu \mathrm{m}$. All solutions were freshly prepared. Temperature and solvent studies were carried out using variable-temperature nano-ESI source, described in detail previously. ${ }^{\mathbf{4 2 , 6 6}}$ Many other electrospray parameters had to be optimized including concentration, size of the emitter tip, distance between the ESI emitter and the inlet of the mass spectrometer, and the applied voltage (see $\mathrm{ESI} \dagger$ for details). Control NMR experiments were run to ensure that the condensation was not taking place in bulk-phase.

\section{On-line analysis}

Online monitoring of reaction intermediates was done using either an ion trap mass spectrometer (LTQ - Velsos, Thermo Fisher, Waltham, MA.) or an ion mobility spectrometry-mass spectrometer (home-built). Variables including the distance between the spray source and the MS-inlet, the inlet temperature, concentration of the reagents, and the reagent flow rate were optimized to maximize product/intermediate abundance (see $\mathrm{ESI} \dagger$ for details).

\section{Off-line analysis}

For the collection of electrosprayed droplets, the spray source was kept close to the collection surface to minimize release of solvent vapor into the air and to maximize droplet collection. Product collection was performed at room temperature on a grounded glass wool surface. Quantitative and structural analysis of the collected spray product (as well as the products of the bulk reactions) was performed using ${ }^{1} \mathrm{H}$ and ${ }^{13} \mathrm{C}$ NMR (see $\mathrm{ESI} \dagger$ for details). Bulk-phase reactions were performed for comparison with reactions occurring in the electro-sprayed droplets.

\section{Conflicts of interest}

There are no conflicts of interest to declare.

\section{Acknowledgements}

We acknowledge funds from Indiana University in partial support of this work. We also gratefully acknowledge the NSF CAREER Award (CHE-1254783). Eli Lilly \& Co. and Amgen supported this work through the Lilly Grantee Award and the Amgen Young Investigator Award. We are also grateful for funds provided by the Robert and Marjorie Mann graduate fellowship (NS).

\section{Notes and references}

1 P. Biginelli, Gazz. Chim. Ital., 1893, 23, 360-416.

2 A. Dömling, W. Wang and K. Wang, Chem. Rev., 2012, 112, 3083-3135.

3 K. S. Atwal, J. L. Bergey, A. Hedberg and S. Moreland, J. Med. Chem., 1987, 30, 635-640.

4 K. S. Atwal, B. C. O'Reilly, E. P. Ruby, C. F. Turk, G. Aberg, M. M. Asaad, J. L. Bergey, S. Moreland and J. R. Powell, J. Med. Chem., 1987, 30, 627-635.

5 L. s. Kürti and B. Czako, Strategic applications of named reactions in organic synthesis : background and detailed mechanisms, Elsevier Academic Press, Amsterdam, Boston, 2005.

6 Â. de Fátima, T. C. Braga, L. d. S. Neto, B. S. Terra, B. G. F. Oliveira, D. L. da Silva and L. V. Modolo, J. Adv. Res., 2015, 6, 363-373.

7 B. L. Nilsson and L. E. Overman, J. Org. Chem., 2006, 71, 7706-7714.

8 F. Cohen and L. E. Overman, J. Am. Chem. Soc., 2001, 123, 10782-10783.

9 D. S. Coffey, L. E. Overman and F. Stappenbeck, J. Am. Chem. Soc., 2000, 122, 4904-4914.

10 L. E. Overman and M. H. Rabinowitz, J. Org. Chem., 1993, 58, 3235-3237.

11 M. M. Heravi, S. Asadi and B. M. Lashkariani, Mol. Diversity, 2013, 17, 389-407.

12 C. O. Kappe, The Biginelli Reaction, in Multicomponent Reactions, ed. J. Zhu and H. Bienaymé, 2005, DOI: 10.1002/ 3527605118.ch4. 
13 Y. Guo, H. Tang, Z. Gao, X. Meng, H. Yu, H. Zhong, G. Huang and C. Zou, ChemistrySelect, 2017, 2, 8253-8255.

14 M. A. Kolosov, D. A. Beloborodov, V. D. Orlov and V. V. Dotsenko, New J. Chem., 2016, 40, 7573-7579.

15 R. Wang and Z.-Q. Liu, J. Org. Chem., 2012, 77, 3952-3958.

16 H. G. O. Alvim, T. B. Lima, A. L. de Oliveira, H. C. B. de Oliveira, F. M. Silva, F. C. Gozzo, R. Y. Souza, W. A. da Silva and B. A. D. Neto, J. Org. Chem., 2014, 79, 3383-3397.

17 Y. Li, Y. Liu, H. Gao, R. Helmy, W. P. Wuelfing, C. J. Welch and R. G. Cooks, Chemistry, 2018, 24, 7349-7353.

18 R. M. Bain, S. T. Ayrton and R. G. Cooks, J. Am. Soc. Mass Spectrom., 2017, 28, 1359-1364.

19 X. Yan, R. M. Bain and R. G. Cooks, Angew. Chem., Int. Ed. Engl., 2016, 55, 12960-12972.

20 R. M. Bain, C. J. Pulliam, S. T. Ayrton, K. Bain and R. G. Cooks, Rapid Commun. Mass Spectrom., 2016, 30, 1875-1878.

21 Y. Li, X. Yan and R. G. Cooks, Angew. Chem., Int. Ed. Engl., 2016, 55, 3433-3437.

22 D. Sarkar, M. K. Mahitha, A. Som, A. Li, M. Wleklinski, R. G. Cooks and T. Pradeep, Adv. Mater., 2016, 28, 22232228.

23 R. M. Bain, C. J. Pulliam and R. G. Cooks, Chem. Sci., 2015, 6, 397-401.

24 R. M. Bain, C. J. Pulliam and R. G. Cooks, Chem. Sci., 2015, 6, 397-401.

25 K. S. Kerian, A. K. Jarmusch and R. G. Cooks, Analyst, 2014, 139, 2714-2720.

26 A. K. Badu-Tawiah, D. I. Campbell and R. G. Cooks, J. Am. Soc. Mass Spectrom., 2012, 23, 1077-1084.

27 X. Yan, Y. H. Lai and R. N. Zare, Chem. Sci., 2018, 9, 52075211.

28 Z. Zhou, X. Yan, Y. H. Lai and R. N. Zare, J. Phys. Chem. Lett., 2018, 9, 2928-2932.

29 J. K. Lee, D. Samanta, H. G. Nam and R. N. Zare, Nat. Commun., 2018, 9, 1562.

30 Y. H. Lai, S. Sathyamoorthi, R. M. Bain and R. N. Zare, J. Am. Soc. Mass Spectrom., 2018, 29, 1036-1043.

31 I. Nam, H. G. Nam and R. N. Zare, Proc. Natl. Acad. Sci. U. S. A., 2018, 115, 36-40.

32 S. Banerjee, E. Gnanamani, X. Yan and R. N. Zare, Analyst, 2017, 142, 1399-1402.

33 Z. Zhou, X. Li and R. N. Zare, ACS Cent. Sci., 2017, 3, 13371344.

34 J. K. Lee, H. G. Nam and R. N. Zare, Q. Rev. Biophys., 2017, 50, e2.

35 I. Nam, J. K. Lee, H. G. Nam and R. N. Zare, Proc. Natl. Acad. Sci. U. S. A., 2017, 114, 12396-12400.

36 R. M. Bain, S. Sathyamoorthi and R. N. Zare, Angew. Chem., Int. Ed. Engl., 2017, 56, 15083-15087.

37 X. Yan, H. Cheng and R. N. Zare, Angew. Chem., Int. Ed. Engl., 2017, 56, 3562-3565.

38 J. K. Lee, S. Banerjee, H. G. Nam and R. N. Zare, Q. Rev. Biophys., 2015, 48, 437-444.

39 J. K. Lee, S. Kim, H. G. Nam and R. N. Zare, Proc. Natl. Acad. Sci. U. S. A., 2015, 112, 3898-3903.

40 Z. Xia and E. R. Williams, Analyst, 2018, 144, 237-248.
41 T. Kenderdine, Z. Xia, E. R. Williams and D. Fabris, Anal. Chem., 2018, 90, 13541-13548.

42 T. J. El-Baba, D. R. Fuller, D. W. Woodall, S. A. Raab, C. R. Conant, J. M. Dilger, Y. Toker, E. R. Williams, D. H. Russell and D. E. Clemmer, Chem. Commun., 2018, 54, 3270-3273.

43 D. N. Mortensen and E. R. Williams, J. Am. Chem. Soc., 2016, 138, 3453-3460.

44 D. N. Mortensen and E. R. Williams, Anal. Chem., 2015, 87, 1281-1287.

45 D. N. Mortensen and E. R. Williams, Anal. Chem., 2014, 86, 9315-9321.

46 D. N. Mortensen and E. R. Williams, Anal. Chem., 2014, 86, 9315-9321.

47 D. E. Clemmer and M. F. Jarrold, J. Mass Spectrom., 1997, 32, 577-592.

48 F. Lanucara, S. W. Holman, C. J. Gray and C. E. Eyers, Nat. Chem., 2014, 6, 281.

49 H. R. Kalita and P. Phukan, Catal. Commun., 2007, 8, 179182.

50 D. Shobha, M. A. Chari, A. Mano, S. T. Selvan, K. Mukkanti and A. Vinu, Tetrahedron, 2009, 65, 10608-10611.

51 P. Li, S. Regati, R. J. Butcher, H. D. Arman, Z. Chen, S. Xiang, B. Chen and C.-G. Zhao, Tetrahedron Lett., 2011, 52, 62206222.

52 H. Murata, H. Ishitani and M. Iwamoto, Org. Biomol. Chem., 2010, 8, 1202-1211.

53 A. Wang, X. Liu, Z. Su and H. Jing, Catal. Sci. Technol., 2014, 4, 71-80.

54 R. Tayebee, M. M. Amini, M. Ghadamgahi and M. Armaghan, J. Mol. Catal. A: Chem., 2013, 366, 266-274.

55 M. Litvić, I. Večenaj, Z. M. Ladišić, M. Lovrić, V. Vinković and M. Filipan-Litvić, Tetrahedron, 2010, 66, 3463-3471.

56 A. Gallo, A. S. F. Farinha, M. Dinis, A.-H. Emwas, A. Santana, R. J. Nielsen, W. A. Goddard and H. Mishra, Chem. Sci., 2019, 10, 2566-2577.

57 M. I. Jacobs, R. D. Davis, R. J. Rapf and K. R. Wilson, J. Am. Soc. Mass Spectrom., 2019, 30, 339-343.

58 D. E. Clemmer, D. H. Russell and E. R. Williams, Acc. Chem. Res., 2017, 50, 556-560.

59 K. Folkers and T. B. Johnson, J. Am. Chem. Soc., 1933, 55, 3784-3791.

60 F. Sweet and J. D. Fissekis, J. Am. Chem. Soc., 1973, 95, 87418749.

61 C. O. Kappe, J. Org. Chem., 1997, 62, 7201-7204.

62 I. Cepanec, M. Litvić, M. Filipan-Litvić and I. Grüngold, Tetrahedron, 2007, 63, 11822-11827.

63 R. O. M. A. De Souza, E. T. da Penha, H. M. S. Milagre, S. J. Garden, P. M. Esteves, M. N. Eberlin and O. A. C. Antunes, Chem.-Eur. J., 2009, 15, 9799-9804.

64 M. M. Gaye, G. Nagy, D. E. Clemmer and N. L. Pohl, Anal. Chem., 2016, 88, 2335-2344.

65 F. Zhu, S. Lee, S. J. Valentine, J. P. Reilly and D. E. Clemmer, J. Am. Soc. Mass Spectrom., 2012, 23, 2158-2166.

66 T. J. El-Baba, D. W. Woodall, S. A. Raab, D. R. Fuller, A. Laganowsky, D. H. Russell and D. E. Clemmer, J. Am. Chem. Soc., 2017, 139, 6306-6309. 